\title{
ON SIMPLE GERMS WITH NON-ISOLATED SINGULARITIES
}

\author{
A. ZAHARIA
}

\section{§1. Introduction.}

Let $\mathcal{O}=\mathcal{O}_{n}$ denote the local ring of germs of analytic functions $f:\left(\mathrm{C}^{n}, 0\right) \rightarrow \mathrm{C}$ and $m$ its maximal ideal. For an analytic germ $f \in \mathcal{O}$ we denote by $J_{f}$ its Jacobi ideal, namely $J_{f}=\left(\frac{\partial f}{\partial z_{1}}, \ldots, \frac{\partial f}{\partial z_{n}}\right)$. For an ideal $I \subset \mathcal{O}$ we consider as in [8], [9]:

- the primitive ideal $\int I$, defined by $\int I=\left\{f \in \mathcal{O} \mid(f)+J_{f} \subset I\right\}$; we have $I^{2} \subset \int I \subset I$

- the group $\mathscr{D}_{I}$ of local analytic isomorphisms $h:\left(\mathrm{C}^{n}, 0\right) \rightarrow\left(\mathrm{C}^{n}, 0\right)$ such that $h^{*}(I)=I$; it is a subgroup of the group of all germs of local analytic isomorphisms of $\left(C^{n}, 0\right)$.

$\mathscr{D}_{I}$ acts on $\int I$ and we shall consider the $\mathscr{R}_{I}$ (right-equivalence) relation on $\int I$. In the next section we prove the following.

THEOREM 1. Let $I \subset \mathcal{O}$ be a radical ideal defining a germ of a quasihomogeneous complete intersection in $\left(\mathrm{C}^{n}, 0\right)$ with isolated singularity. Suppose that there exist $\mathscr{R}_{I}$-simple germs in $\int I$. Then in some coordinates $\left(z_{1}, \ldots, z_{n}\right)$ of $\left(\mathrm{C}^{n}, 0\right)$ we have either

a) there exists $k \in\{1, \ldots, n\}$ such that $I=\left(z_{1}, \ldots, z_{k}\right)$, or

b) there exists $k \in\{1, \ldots, n\}$ and a quasihomogeneous isolated singularity $g=g\left(z_{1}, \ldots, z_{k}\right) \in \mathcal{O}_{k}$ such that $I=\left(g, z_{k+1}, \ldots, z_{n}\right)$.

A. Némethi has proved a similar result in [7] for the case when $I=\left(f^{s}\right)$ where $s \geqq 1$ and $f \in \mathcal{O}$ is an isolated singularity. When $n=3$, D. Siersma has considered a similar problem for the inner modality (see [12]).

In the last section we derive the list of $\mathscr{R}_{1}$-simple germs for $I=\left(z_{1}, z_{2}\right)$.

\section{§2. Proof of Theorem 1.}

We recall from [8], [9] that for an ideal $I \subset \mathcal{O}$ and for $f \in \int I$, the tangent space at 
$f$ to the $\mathscr{R}_{I}$-orbit of $f$ is defined by

$$
T_{I}(f)=\left\{\eta(f) \mid \eta=\sum_{j=1}^{n} \eta_{j} \frac{\partial}{\partial z_{j}} \text { with } \eta(I) \subset I \text { and } \eta_{j} \in m \text { for } j=1, \ldots, n\right\}
$$

and the $I$-codimension of $f$ is

$$
c_{I}(f)=\operatorname{dim}_{C} \frac{\int I}{T_{I}(f)} .
$$

Let $f_{1}, \ldots, f_{p}$ be a minimal set of quasihomogeneous generators of $I$. Let $q$ be the dimension of the $\mathrm{C}$-vector space $\left(I+m^{2}\right) / m^{2}$. If $q=p$, we have a) with $k=q=p$.

Suppose that $q<p$. Using a linear change of coordinates, we can assume, without altering the quasihomogeneity of $f_{1}, \ldots, f_{p}$, that $f_{j}(z)=z_{j}+$ higher monomials not containing $z_{j}$, for $j=1, \ldots, q$ (we assume that the weights of the coordinates are positive). Thus, we can consider, by subtracting suitable multiples of $f_{1}, \ldots, f_{q}$, if necessary, that $f_{q+1}, \ldots, f_{p}$ are quasihomogeneous polynomials, not depending on $z_{1}, \ldots, z_{q}$. It follows that, in a suitable system $z$ of coordinates, the ideal $I$ is generated by $f_{1}=z_{1}, \ldots, f_{q}=z_{q}, f_{q}=z_{q}, f_{q+1}, \ldots, f_{p}$, where $f_{q+1}, \ldots, f_{p} \in m^{2}$ are quasihomogeneous polynomials depending only on $z_{q+1}, \ldots, z_{n}$.

Since there exist $\mathscr{R}_{I}$-simple germs in $\int I$, we can find $f \in \int I$ such that $c_{I}(f)=0$. (The $\mathscr{R}_{\Gamma}$-simple germs are defined similarly with the simple isolated singularities; see for example [2] or [4].) From [8], [9], we have $\int I=I^{2}$ and we can write $f=\sum_{i, j=1}^{p} g_{i j} f_{i} f_{j}$, with $g_{i j}=g_{j i}$. Let $r$ be the rank of the matrix $\left(g_{i j}(0)\right)_{i, j=1, q}$. Then $r$ is also the rank of the Hessian matrix evaluated in $0,\left(\frac{\partial^{2} f}{\partial z_{i} \partial z_{j}}(0)\right)_{i, j=1, n}$. As in the proof of Morse Lemma (see for example [6]) we can obtain a system $\tilde{z}$ of coordinates, with $\tilde{z}_{j}=z_{j}$ for $j>q$, such that $I$ is generated by $\tilde{f}_{1}=\tilde{z}, \ldots, \tilde{f}_{q}=\tilde{z}_{q}$, $\tilde{f}_{q+1}=f_{q+1}, \ldots, \tilde{f}_{p}=f_{p}$ and such that

$$
f=\tilde{z}_{1}^{2}+\ldots+\tilde{z}_{r}^{2}+\sum_{i, j=r+1}^{p} \tilde{g}_{i j} \tilde{f}_{i} \tilde{f}_{j},
$$

with $\tilde{g}_{i j}=\tilde{g}_{j i}$ and with $\tilde{g}_{i j} \tilde{f}_{i} \tilde{f}_{j} \in m^{3}$. It is easy to see that for any $i, j \geqq r+1$, there exists $h_{i j}=h_{i j}\left(\tilde{z}_{r+1}, \ldots, \tilde{z}_{n}\right)$ with $h_{i j} \tilde{f}_{i} \tilde{f}_{j} \in m^{3}$ and such that for any $k \in \mathrm{N}, k \geqq 2$, we have that $f$ is $\mathscr{R}_{I}$-equivalent to $\tilde{z}_{1}^{2}+\ldots+\tilde{z}_{r}^{2}+\sum_{i, j=r+1}^{p} h_{i j} \tilde{f}_{i} \tilde{f}_{j}+\sum_{i, j=r+1}^{p} \varphi_{i j} f_{i} f_{j}$, for some $\varphi_{i j} \in\left(\tilde{z}_{1}, \ldots, \tilde{z}_{r}\right)^{k}$. Since $c_{I}(f)<\infty, f$ is $I$-finitely determined (see [8], [9]). Hence we can assume that in (1) the germs $\tilde{g}_{i j}$ do not depend on $\tilde{z}_{1}, \ldots, \tilde{z}_{r}$.

We shall write in the sequel $z$ for $\tilde{z}, f_{j}$ for $\widetilde{f}_{j}$ and $g_{i j}$ for $\tilde{g}_{i j}$. Since $c_{I}(f)=0$, we 
must have $T_{I}(f)=\int I=I^{2}$; we prove that this equality implies that $r=q=p-1$.

Let $\theta_{I}=\left\{\eta=\sum_{j=1}^{n} \eta_{j} \frac{\partial}{\partial z_{j}} \mid \eta(I) \subset I\right\}$ be the $\mathcal{O}$-module of logarithmic vector fields for $I$. Since $I=\left(f_{1}, \ldots, f_{p}\right)$ is a reduced quasihomogeneous complete intersection in $\left(\mathrm{C}^{n}, 0\right)$ with isolated singularity, the $\mathcal{O}$-module $\theta_{I}$ is generated by the following vector fields (see for example [3]):

(A) $f_{i} \frac{\partial}{\partial z_{j}}$, where $i=1, \ldots, p$ and $j=1, \ldots, n$;

(B) the "trivial vector fields"

$$
\left|\begin{array}{ccc}
\frac{\partial}{\partial z_{i_{1}}} & \ldots & \frac{\partial}{\partial z_{i_{p+1}}} \\
\frac{\partial f_{1}}{\partial z_{i_{1}}} & \ldots & \frac{\partial f_{1}}{\partial z_{i_{p+1}}} \\
\ldots \ldots \ldots \ldots \ldots \ldots \\
\frac{\partial f_{p}}{\partial z_{i_{1}}} \ldots & \frac{\partial f_{p}}{\partial z_{i_{p+1}}}
\end{array}\right|
$$

for all $(p+1)$-tuples $\left(i_{1}, \ldots, i_{p+1}\right)$ satisfying $1 \leqq i_{1} \leqq i_{2} \leqq \ldots \leqq i_{p+1} \leqq n$;

(C) the Euler vector field $E=\sum_{j=1}^{n} w_{j} z_{j} \frac{\partial}{\partial z_{j}}$, where $w_{1}, \ldots, w_{n}$ are the weights of the coordinates.

It is clear that $T_{I}(f)=\theta_{I}(f)$.

We recall that $f_{j}=z_{j}$ for $1 \leqq j \leqq q$ and $f_{q+1}, \ldots, f_{p} \in m^{2}$ do not depend on $z_{1}, \ldots, z_{q}$. Also we recall that $f=z_{1}^{2}+\ldots+z_{r}^{2}+\sum_{i, j=r+1}^{p} g_{i j} f_{i} f_{j}$ with $g_{i j}=g_{j i}$ not depending on $z_{1}, \ldots, z_{r}$ and with $g_{i j} f_{i} f_{j} \in m^{3}$.

Suppose first that $r<q$. Then a moment's thought will convince us that for any $\eta \in \theta_{I}$, if we consider the expansion of $\eta(f)$ in a power series, then the coefficient of $z_{q}^{2}$ is zero. Hence $z_{q}^{2} \notin T_{I}(f)=I^{2}$, a contradiction. It follows that $r=q$.

We look now for $f_{q+1}^{2}, \ldots, f_{p}^{2}$. It is easy to see that if $\eta \in \theta_{I}$ is one of the generators from (A) or (B), then $\eta(f)$ belongs to the ideal $L=m \cdot\left(f_{q+1}, \ldots, f_{p}\right)^{2}+$ $\left(z_{1}, \ldots, z_{q}\right) \cdot\left(f_{q+1}, \ldots, f_{p}\right)+\left(z_{1}, \ldots, z_{q}\right)^{2}$. On the other hand, for any germ $g \in m$ we have also $(g E)(f) \in L$. Thus $\theta_{I}(f)=L+\mathrm{C} \cdot E(f)$. If $p-q \geqq 2$ we have the uniqueness of the weights $w_{q+1}, \ldots, w_{n}$ (see for example [4]), hence $f_{q+1}^{2}, \ldots, f_{p}^{2}$ can not belong simultaneously to $\theta_{I}(f)$, in contradiction with the equality $I^{2}=\theta_{I}(f)$. It follows that $q+1=p$. The theorem is proved. 


\section{§3. The simple germs for $I=\left(z_{1}, z_{2}\right)$.}

D. Siersma has found the $\mathscr{R}_{I}$-simple germs when $I=\left(z_{1}, \ldots, z_{n-1}\right) \subset \mathcal{O}$ in [10] and for $I=\left(z_{1} z_{2}, z_{3}, \ldots, z_{n}\right) \subset \mathcal{O}$ in [12]. For the case when $I=\left(z_{1}\right) \subset \mathcal{O}$, the list of $\mathscr{R}_{I}$-simple germs follows from the work of V.I. Arnold [1] (see for example [13]).

In the sequel we derive the list of $\mathscr{R}_{I}$-simple germs for $I=\left(z_{1}, z_{2}\right)$. We shall suppose that $n \geqq 4$ and we shall consider only germs $f \in I^{2}$ with $j^{2} f=0$. (The simple germs $f \in I^{2}$ with $j^{2} f \neq 0$ are suspensions of those in [13].)

We use the following classical lemma:

LEMMA. Let $f_{t}=f+t \cdot \phi \in I^{2}$ be a family of germs, with $t \in \mathrm{R}$.

a) If $\phi \in \mathscr{T}\left(f_{t}\right)$ for every $t \in R$, then, for any $t \in R, f_{t}$ is $\mathscr{R}_{I}$-equivalent with $f_{0}$.

b) If $\phi \notin \mathscr{T}\left(f_{t}\right)$ for every $t \in \mathrm{R}$, then, for any $t \in \mathrm{R}, f_{t}$ is not $\mathscr{R}_{I}$-simple.

If we denote the coordinates $z_{3}, \ldots, z_{n}$ by $u_{1}, \ldots, u_{n-2}$ and the Milnor number of an isolated singularity $g$ by $\mu(g)$, we have the following:

THEOREM 2. Let $I=\left(z_{1}, z_{2}\right) \subset \mathcal{O}$ and $f \in I^{2}$ with $j^{2} f=0$. Then $f$ is $\mathscr{R}_{I}$-simple if and only if $f$ is $\mathscr{R}_{I}$-equivalent to a germ in the following table.

\begin{tabular}{|c|c|c|c|}
\cline { 2 - 4 } \multicolumn{1}{c|}{} & Normal form of $f$ & $c_{I}(f)$ & Conditions \\
\hline$I_{n}$ & $u_{1} z_{1}^{2}+u_{2} z_{2}^{2}+u_{3} z_{1} z_{2}$ & 3 & $n \geqq 5$ \\
\hline I4 & $u_{1} z_{1}^{2}+u_{2} z_{2}^{2}$ & 3 & $n=4$ \\
\hline II & $u_{1} z_{1}^{2}+u_{2} z_{2}^{2}+z_{1} z_{2} \cdot g\left(u_{3}, \ldots, u_{n-2}\right)$ & $n-2+\mu(g)$ & $n \geqq 5 ; g \in \mathrm{A}-\mathrm{D}-\mathrm{E}$ \\
\hline III & $u_{1} z_{1} z_{2}+u_{2} z_{1}^{2}+z_{2}^{2}\left(z_{2}+u_{2}^{k}+u_{3}^{2}+\ldots+u_{n-2}^{2}\right)$ & $k+n-2$ & $n \geqq 4 ; k \geqq 2$ \\
\hline & $u_{1} z_{1} z_{2}+u_{2} z_{1}^{2}+z_{2}^{2}\left(z_{2}+u_{2} u_{3}+u_{3}^{k}+u_{4}^{2}+\ldots+u_{n-2}^{2}\right)$ & $k+n-2$ & $n \geqq 5 ; k \geqq 3$ \\
\hline & $u_{1} z_{1} z_{2}+u_{2} z_{1}^{2}+z_{2}^{2}\left(z_{2}+u_{2}^{2}+u_{3}^{3}+u_{4}^{2}+\ldots+u_{n-2}^{2}\right)$ & $n+2$ & $n \geqq 5$ \\
\hline IV & $u_{1} z_{1} z_{2}+u_{2} z_{1}^{2}+z_{2}^{2}\left(z_{2}^{k}+u_{2}^{2}+\ldots+u_{n-2}^{2}\right)$ & $k+n-1$ & $n \geqq 4 ; k \geqq 2$ \\
\hline Va & $u_{1} z_{1} z_{2}+u_{2} z_{1}^{2}+z_{2}^{2}\left(z_{2}^{2}+u_{2}^{3}+u_{3}^{2}+\ldots+u_{n-2}^{2}\right)$ & $n+3$ & $n \geqq 4$ \\
\hline Vb & $u_{1} z_{1} z_{2}+u_{3} z_{1}^{2}+z_{2}^{2}\left(z_{2}^{2}+u_{2}^{3}+u_{3}^{2}+\ldots+u_{n-2}^{2}\right)$ & $n+4$ & $n \geqq 5$ \\
\hline VI & $u_{1} z_{1} z_{2}+u_{2} z_{1}^{2}+z_{2}^{2}\left(z_{2} u_{2}+u_{2}^{k}+u_{3}^{2}+\ldots+u_{n-2}^{2}\right)$ & $k+n-1$ & $n \geqq 4 ; k \geqq 3$ \\
\hline VI & $u_{1} z_{1} z_{2}+u_{3} z_{1}^{2}+z_{2}^{2}\left(z_{2} u_{2}+u_{2}^{3}+u_{3}^{2}+\ldots+u_{n-2}^{2}\right)$ & $n+4$ & $n \geqq 5$ \\
\hline
\end{tabular}

PROOF: If $f \in I^{2}$ has $j^{2} f=0$, then $j^{3} f=u_{1} Q_{1}\left(z_{1}, z_{2}\right)+\ldots+u_{n-2} Q_{n-2}\left(z_{1}, z_{2}\right)$ $+C\left(z_{1}, z_{2}\right)$, where $Q_{1}, \ldots, Q_{n-2}$ are quadrics and $C$ is a cubic in $z_{1}, z_{2}$. We suppose that $c_{I}(f)<\infty$. Hence $f$ is $\mathscr{R}_{I}$-equivalent to a jet $j^{k} f$ for sufficiently large $k$ (see [9]). 
Let $V$ be the C-vector space generated by $Q_{1}, \ldots, Q_{n-2}$ in the vector space of quadrics in $z_{1}, z_{2}$.

If $\operatorname{dim} V=3$ then $n \geqq 5$ and we can find in $\mathscr{D}_{I}$ a linear isomorphismm of $\left(C^{n}, 0\right)$ such that $j^{3} f=u_{1} z_{1}^{2}+u_{2} z_{2}^{2}+u_{3} z_{1} z_{2}$. It follows by [8], [9] that $f$ is $\mathscr{R}_{I}$-equivalent with $j^{3} f\left(f\right.$ is a $D(1,1)$-type germ) and $f$ is $\mathscr{R}_{I}$-simple.

If $\operatorname{dim} V \leqq 1$ then $f$ is not $\mathscr{R}_{I}$-simple. Namely, any neighbourhood of $f$ contains a germ which is $\mathscr{R}_{1}$-equivalent to a germ $f=u_{1} z_{1} z_{2}+z_{1}^{3}+$ $z_{2}^{3}+z_{1}^{2}\left(u_{2}^{2}+\ldots+u_{n-2}^{2}\right)+z_{2}^{2} \cdot \varphi\left(u_{2}, \ldots, u_{n-2}\right)$ where $\varphi \in m^{2}$. It is easy to see that for any $\varphi, \tilde{f}$ is not $\mathscr{R}_{I}$-simple.

If $\operatorname{dim} V=2$ then, using the classification of pencils of quadrics in $z_{1}, z_{2}$ we can find in $\mathscr{D}_{I}$ some linear isomorphisms of $\left(\mathbf{C}^{n}, 0\right)$ such that $j^{3} f$ is one of the following cubics:

$$
u_{1} z_{1}^{2}+u_{2} z_{2}^{2} ; u_{1} z_{1} z_{2}+u_{2} z_{1}^{2} \text { or } u_{1} z_{1} z_{2}+u_{2} z_{1}^{2}+z_{2}^{3} .
$$

When $j^{3} f=u_{1} z_{1}^{2}+u_{2} z_{2}^{2}$ it follows, directly or using the technique of global transversal from [5], that $f$ is $\mathscr{R}_{I}$-equivalent to $u_{1} z_{1}^{2}+u_{2} z_{2}^{2}+z_{1} z_{2} g\left(u_{3}, \ldots\right.$, $\left.u_{n-2}\right)$. Now it is easy to see, for $n \geqq 5$, that $f$ is $\mathscr{R}_{I}$-simple if and only if $g$ is a simple isolated singularity ( $g$ is an A-D-E singularity; see [2], or [4] for the normal forms).

If $j^{3} f=u_{1} z_{1} z_{2}+u_{2} z_{1}^{2}+z_{2}^{3}$, then $f$ is $\mathscr{R}_{1}$-equivalent to $u_{1} z_{1} z_{2}+u_{2} z_{1}^{2}+$ $z_{2}^{2}\left(z_{2}+g\left(u_{2}, \ldots, u_{n-2}\right)\right)$ with $g \in m^{2}$. It is easy to see that $f$ is $\mathscr{R}_{I}$-simple if and only if $g$ is a simple boundary singularity in the sense of Arnold, the boundary being $u_{2}=0$ (see [1]).

The most difficult case is when $j^{3} f=u_{1} z_{1} z_{2}+u_{2} z_{1}^{2}$. In this situation $f$ is $\mathscr{R}_{I}$-equivalent to $u_{1} z_{1} z_{2}+u_{2} z_{1}^{2}+z_{2}^{2} h\left(z_{2}, u_{2}, \ldots, u_{n-2}\right)$ with $h \in m^{2}$. If $h$ is a simple boundary singularity with respect to $z_{2}=0$, we change the coordinates such that $h$ becomes the normal form of a B-C-F singularity. Then $f$ is $\mathscr{R}_{r}$-equivalent to $u_{1} z_{1} z_{2}+\varphi\left(u_{2}, \ldots, u_{n-2}\right) z_{1}^{2}+z_{2}^{2} h$, with $\varphi \in m \backslash m^{2}$, and we obtain the germs in the table by using the lemma and loocking at $j^{1} \varphi$.

If $h$ is not a simple boundary singularity then $f$ can be deformed to a germ which is $\mathscr{R}_{r}$-equivalent to $u_{1} z_{1} z_{2}+\varphi\left(u_{2}, \ldots, u_{n-2}\right) z_{1}^{2}+z_{2}^{2} h\left(z_{2}, u_{2}, \ldots, u_{n-2}\right)$ where $\varphi \in m \backslash m^{2}$ and $h$ is one of the following unimodal boundary singularities (see [2]):

or

$$
\begin{aligned}
& F_{1,0}: z_{2}^{3}+a z_{2} u_{2}^{2}+u_{2}^{3}+u_{3}^{2}+\ldots+u_{n-2}^{2}, 4 a^{3}+27 \neq 0 \\
& K_{4,2}: z_{2}^{2}+a z_{2} u_{2}^{2}+u_{2}^{4}+u_{3}^{2}+\ldots+u_{n-2}^{2}, a^{2} \neq 4
\end{aligned}
$$$$
L_{6}: z_{2} u_{2}+a z_{2} u_{3}+u_{2}^{2} u_{3}+u_{3}^{3}+u_{4}^{2}+\ldots+u_{n-2}^{2} \text {. }
$$

Using the lemma with $\mathscr{T}(f)$ replaced by $\mathscr{T}(f)+\left(u_{2}, \ldots, u_{n-2}\right)^{3}\left(z_{1}\right)^{2}$ we obtain that $f$ is not $\mathscr{R}_{I}$-simple. 


\title{
REFERENCES
}

1. V. I. Arnold, Critical points of functions on a manifold with boundary, the simple Lie groups $B_{k}, C_{k}$ and $F_{4}$ and singularities of evolutes, Uspekhi Mat. Nauk 33 (1978), 91-105.

2. V. I. Arnold, S. M. Gusein-Zade and A. N. Varchenko, Singularities of Differentiable Maps I, Monograph Math. 82, Birkhäuser, Boston-Basel-Stuttgart, 1985.

3. J. W. Bruce and R. M. Roberts, Critical points of functions on analytic varieties, Topology 27 (1988), 57-90.

4. A. Dimca, Topics on Real and Complex Singularities, Vieweg Verlag Braunschweig, 1987.

5. A. Dimca and C. G. Gibson, Classification of equidimensional contact unimodular map germs, Math. Scand. 56 (1985), 15-28.

6. J. Milnor, Morse Theory, Ann. of Math. Studies 51, Princeton University Press, 1963.

7. A. Némethi, The Milnor fibr and the zeta function of the singularities of type $f=P(h, g)$, Compositio Math.

8. G. R. Pellikaan, Hypersurfaces singularities and Resolutions of Jacobi Modules, Thesis, Rijksuniversiteit Utrecht, 1985.

9. G. R. Pellikaan, Finite determinacy of functions with non-isolated singularities, Proc. London Math. Soc. 57 (1988), 357-382.

10. D. Siersma, Isolated line singularities, Proceedings Sympos. Pure Math. 40 (1983), 485-496.

11. D. Siersma, Hypersurfaces with singular locus a plane curve and transversal type $\mathrm{A}_{1}$, Singularities Banach Center Publications 20, PWN-Polish Scientific Publishers Warsaw (1988), 397-410.

12. D.Siersma, Quasihomogeneous singularities with transversal type $\mathrm{A}_{1}$, Contemp. Math. 90 (1989), 261-294.

13. A. Zaharia, Sur une classe de singularités non-isolées, Rev. Roum. Math. Pures Appl. 35 (1990), 373-378.

\author{
DEPARTMENT OF MATHEMATICS \\ INCREST \\ BD. PACII 220 \\ 79622 BUCHAREST \\ ROUMANIA
}

